\title{
Holomorphic Extension from Boundaries with Concentrated Levi Form
}

\author{
DAVID E. BARRETT
}

We consider the problem of identifying boundary values of holomorphic functions on (non-smooth) bounded domains in $\mathbb{C}^{2}$ with boundaries that are Levi-flat off of a small set.

Assuming also that the domain is circular, we construct a type of extension operator from the algebra of bounded holomorphic functions on a leaf of the induced Levi-foliation to the algebra of bounded holomorphic functions on the domain itself, thereby obtaining another proof of the result in $[\mathrm{BaDi}]$ to the effect that such leaves have non-empty corona.

1. Introduction. Let $\Omega$ be a bounded domain in $\mathbb{C}^{2}$ containing the origin. We make the standing assumptions:

(1) for each $\zeta \in \hat{\mathbb{C}} \stackrel{\text { def }}{=} \mathbb{C} \cup\{\infty\}$ the slice $\Omega_{\zeta} \stackrel{\text { def }}{=}\left\{z \in \Omega: z_{1} / z_{2}=\zeta\right\}$ is a simply-connected domain bounded by a Jordan curve;

(2) $\sup _{\zeta} \Lambda_{1}\left(\mathrm{~b} \Omega_{\zeta}\right)<\infty$.

Here $\Lambda_{1}$ denotes one-dimensional Hausdorff measure.

Recall that a smooth real hypersurface is said to be Levi-flat if its Levi form vanishes identically; this is equivalent to saying that the real hypersurface is foliated by codimension-one complex hypersurfaces.

Let

$$
P=\left\{z \in \mathrm{b} \Omega: \mathrm{b} \Omega \text { is } C^{2} \text {-smooth and Levi-flat near } z\right\}
$$

and

$$
K=\left\{z_{1} / z_{2}: z \in \mathrm{b} \Omega \backslash P\right\} \subset \hat{\mathbb{C}} .
$$


Also let

$$
\begin{gathered}
A(\Omega)=\{F \in C(\bar{\Omega}): F \text { holomorphic on } \Omega\}, \\
H^{\infty}(\Omega)=\left\{F \in L^{\infty}(\Omega): F \text { holomorphic }\right\}, \\
A(P)=\{f \in C(\mathrm{~b} \Omega): f \text { is holomorphic along leaves of } P\},
\end{gathered}
$$

and

$$
H^{\infty}(P)=\frac{\{f \text { bounded and measurable on } P \text { and holomorphic along leaves }\}}{\{f \text { vanishing identically on almost every leaf }\}} .
$$

Note that each $f \in H^{\infty}(P)$ is defined almost everywhere on $\mathrm{b} \Omega_{\zeta}$ for each $\zeta \notin K$.

Theorem 1.1 Suppose that

(1) $\Lambda_{1}(K)<\infty$;

(2) the map $\zeta \mapsto b \Omega_{\zeta}$ from $\hat{\mathbb{C}}$ into the space of 1 -currents on $\mathbb{C}^{2}$ is continuous.

Then each $f \in A(P)$ extends to a function $F \in A(\Omega)$.

Theorem 1.2 Suppose that $\Lambda_{1}(K)=0$. Then each $f \in H^{\infty}(P)$ extends to a function $F \in H^{\infty}(\Omega)$ with boundary values $f$.

See [Mor, Chap. 4] and [Fal, Chap. 3] for background connected with condition (2) of Theorem 1.1. The boundary values in Theorem 1.2 may be taken non-tangentially almost everywhere along each $\mathrm{b} \Omega_{\zeta}, \zeta \notin K$.

Examples of domains $\Omega$ satisfying the hypotheses of these two theorems are discussed in Section 2. Proofs of the theorems are provided in Section 3.

We turn now to questions connected with leaves of the Levi-foliation of $P$.

Theorem 1.3 Suppose that $\Lambda_{1}(K)=0$. Then no leaf of the Levi-foliation of $P$ is closed in $P$.

Theorem 1.4 Suppose that $\Omega$ is circular (i.e., each $\Omega_{\zeta}$ is a disk centered at 0$)$ and $\Lambda_{1}(K)=0$. Let $L$ be a leaf of the Levi-foliation on $P$ and let $H^{\infty}(L)$ denote the algebra of bounded holomorphic functions on $L$. Then there is an operator

$$
E: H^{\infty}(L) \rightarrow H^{\infty}(\Omega)
$$

such that

(1) $E\left(\left.F\right|_{L}\right)=f$ for all $F \in A(\Omega)$; 
(2) $\|E\|=1$;

(3) $E(f g)=E(f) \cdot E(g)$.

We do not know if the conditions in the statement of 1.4 determine $E$ uniquely.

The map $E$ induces a map

$$
\hat{E}: \operatorname{Spec} H^{\infty}(\Omega) \rightarrow \operatorname{Spec} H^{\infty}(L)
$$

of maximal ideal spaces. Letting

$$
\text { ८. : } \rightarrow \operatorname{Spec} H^{\infty}(\cdot)
$$

denote the natural inclusion, it is easy to check that $\hat{E}\left(\iota_{\Omega}(\Omega)\right)$ is disjoint from the closure of $\iota_{L}(L)$. Thus we obtain a new proof of the following result of the author and Jeff Diller [BaDi].

Theorem 1.5 Under the hypotheses of Theorem 1.4, the corona

$$
\operatorname{Spec} H^{\infty}(L) \backslash \overline{\iota_{L}(L)}
$$

is non-empty.

The proofs of Theorems 1.3, 1.4, and 1.5 appear in Section 4 below.

\section{Examples.}

Example 2.1 (Bidisk) Let $\Delta$ denote the unit disk in $\mathbb{C}$, let $\mathbb{T}$ denote the unit circle $\mathrm{b} \Delta$, and consider the bidisk $\Omega=\Delta \times \Delta$.

We have

$$
\begin{gathered}
P=(\Delta \times \mathbb{T}) \cup(\mathbb{T} \times \Delta), \\
K=\{\zeta \in \hat{\mathbb{C}}:|\zeta|=1\} .
\end{gathered}
$$

Theorem 1.1 is easily seen to apply and in particular we recover the well-known fact that a continuous function $f$ on the distinguished boundary $\mathbb{T} \times \mathbb{T}$ extends to $A(\Omega)$ if and only if $f$ extends holomorphically to each of the disks $\{\cdot\} \times \Delta, \Delta \times$ $\{\cdot\}$ in $P$.

Example 2.2 (Circular domains) More generally, let $\Omega \Subset \mathbb{C}^{2}$ be a complete circular domain (i.e., $|\lambda|<1$ implies $\lambda \Omega \subset \Omega$ ) and let $r(\zeta)$ be the radius of $\Omega$ in the complex direction $\zeta$ :

$$
r(\zeta) \stackrel{\text { def }}{=} \sup \left\{\|z\|: \frac{z_{1}}{z_{2}}=\zeta\right\} .
$$


Then $\log r$ is a bounded lower semi-continuous function on $\hat{\mathbb{C}}$.

Let

$$
\omega=\frac{2 i d \zeta \wedge d \bar{\zeta}}{\left(|\zeta|^{2}+1\right)^{2}}=d * d \log \sqrt{|\zeta|^{2}+1},
$$

the spherical area form on $\hat{\mathbb{C}}$.

Proposition 2.3 $K=\operatorname{supp}(\omega-d * d \log r) \cup \overline{\{\zeta: r \text { discontinuous at } \zeta\}}$.

See also [HuPa, Section 7], [Sib].

Proof. Since all terms of the statement of the Proposition transform appropriately under unitary transformations of $\mathbb{C}^{2}$, it will suffice to work near $\zeta_{0} \neq \infty$.

If $\zeta_{0} \notin K$, then there is a germ at $\zeta_{0}$ of a complex curve

$$
\zeta \mapsto H(\zeta)=\left(h_{1}(\zeta), h_{2}(\zeta)\right)
$$

in $\mathrm{b} \Omega$ with $\frac{h_{1}(\zeta)}{h_{2}(\zeta)} \equiv \zeta$. Thus $r(\zeta)=\|H(\zeta)\|$ is continuous near $\zeta$ and

$$
\begin{aligned}
d * d \log r & =d * d \log \|H(\zeta)\| \\
& =d * d \log \left|h_{2}(\zeta)\right|+d * d \log \sqrt{|\zeta|^{2}+1} \\
& =\omega
\end{aligned}
$$

near $\zeta_{0}$.

On the other hand, if $d * d \log r \equiv \omega$ near $\zeta_{0}$ (in the sense of distributions), then

$$
d * d \log \left(\frac{r}{\sqrt{|\zeta|^{2}+1}}\right) \equiv 0
$$

near $\zeta_{0}$ so that $\log \left(r /\left(\sqrt{|\zeta|^{2}+1}\right)\right)$ agrees almost everywhere with a harmonic function $u$ in a neighborhood of $\zeta_{0}$. If $r$ is continuous near $\zeta$, then, in fact, $\log \left(r /\left(\sqrt{|\zeta|^{2}+1}\right)\right)$ coincides with $u$ near $\zeta_{0}$. Picking a local harmonic conjugate $v$ for $u$, the complex curves

$$
\zeta \mapsto\left(\zeta e^{u(\zeta)+i(v(\zeta)+C)}, e^{u(\zeta)+i(v(\zeta)+C)}\right)
$$

provide the requisite Levi-foliation of $\mathrm{b} \Omega$ near $\mathrm{b} \Omega_{\zeta_{0}}$ showing that $\zeta_{0} \notin K$.

The current $\omega-d * d \log r$ is of course just a suitably normalized version of the Levi form. Note that

$$
\int_{\widehat{\mathbb{C}}}(\omega-d * d \log r)=\int_{\widehat{\mathbb{C}}} \omega=4 \pi
$$


hence $K \neq \varnothing$. Thus by using unitary invariance as in the proof of Proposition 2.3 , we may and shall assume below that $\infty \in K$.

If $L \subset \hat{\mathbb{C}}$ is a compact set of positive logarithmic capacity (in particular, if $L$ has positive Hausdorff dimension), then there is a continuous function $r$ on $\hat{\mathbb{C}}$ smooth off of $L$ and satisfying $\omega-d * d \log r \geq 0, \operatorname{supp}(\omega-d * d \log r) \subset L$. Letting $\Omega$ be the corresponding circular domain, we find that $K \subset L$. If we choose $L$ so that $\Lambda_{1}(L)<\infty$, then $\Omega$ satisfies the hypotheses of Theorem 1.1; if $\Lambda_{1}(L)=0$ then $\Omega$ satisfies the hypotheses of Theorem 1.2. (See [Tsu, Thms. III.12, III.19], [Car, Section III.1].)

We include a few remarks on the monodromy of the Levi-foliation of $P$ which will be used in Section 4 .

For a planar domain (or Riemann surface) $X$, we let $\rho_{\mathrm{Hom}}^{X}: \widetilde{X}_{\mathrm{Hom}} \rightarrow X$ denote the homology covering of $X$. This is the covering space of $X$ corresponding to the commutator subgroup $\pi_{1}^{\prime}(X)$ of $\pi_{1}(X)$. Since $\pi_{1}^{\prime}(X)$ is a normal subgroup of $\pi_{1}(X), \widetilde{X}_{\text {Hom }}$ is a regular covering of $X$ with deck group $\pi_{1}(X) / \pi_{1}^{\prime}(X)=H^{1}(X)$. If $X$ is open, then $H^{1}(X)$ is the smallest covering space of $X$ on which we may define conjugates of arbitrary harmonic functions on $X$ [For, 28.6].

Thus, in particular, if $D$ is any component of $\hat{\mathbb{C}} \backslash K$, then the harmonic conjugate $v$ used in the proof of Proposition 2.3 is single-valued on $\widetilde{D}_{\mathrm{Hom}}$. Hence we have a CR covering map

$$
\begin{aligned}
\widetilde{D}_{\mathrm{Hom}} \times \mathbb{T} & \rightarrow P \\
(\xi, \tau) & \mapsto\left(\tau \rho_{\mathrm{Hom}}^{X}(\xi) e^{u\left(\rho_{\mathrm{Hom}}^{X}(\xi)\right)+i v(\xi)}, \tau e^{u\left(\rho_{\mathrm{Hom}}^{X}(\xi)\right)+i v(\xi)}\right) ;
\end{aligned}
$$

the deck group can be identified with $H^{1}(D)$ via the action

$$
\gamma \cdot(\xi, \tau)=(\gamma \cdot \xi, \chi(\gamma) \cdot \tau)
$$

where

$$
\begin{aligned}
\chi(\gamma) & =e^{i(v(\gamma \cdot \xi)-v(\xi))} \\
& =e^{i \int_{\gamma} * d u} .
\end{aligned}
$$

Thus, in particular, continuation of a leaf $L$ of $P$ along a loop $\gamma$ in $D$ rotates $L$ by $\chi(\gamma)$.

The monodromy map

$$
\chi: H^{1}(D) \rightarrow \mathbb{T}
$$

is closely related to the Levi current $\omega-d * d \log r$ since for any loop $\gamma$ in $D$ 
bounding a domain $V \subset \mathbb{C}$ we have

$$
\begin{aligned}
\int_{\gamma} * d u & =\int_{\gamma} * d \log \left(\frac{r}{\sqrt{|\zeta|^{2}+1}}\right) \\
& =\int_{V} d * d \log \left(\frac{r}{\sqrt{|\zeta|^{2}+1}}\right) \\
& =-\int_{V}(\omega-d * d \log r)
\end{aligned}
$$

so that

$$
\chi(\gamma)=e^{-i(\text { Levi measure of } V)} .
$$

Remarks The preceding equation reflects the $G L(2, \mathbb{C})$-invariance of our normalization of the Levi form. This may be contrasted with the normalization used, for example, in $[\mathrm{BG}]$, which is invariant under the affine-unitary group.

For higher-dimensional circular domains the current $\omega-d * d \log r$ should be replaced by the difference of the Fubini-Study Kähler form on $\mathbb{C} P^{n-1}$ and the form $2 i \partial \bar{\partial} \log r$; see $[\mathrm{HuPa}]$ for additional information.

Example 2.4 (Homogeneous superattracting basins) Let $\Phi: \mathbb{C}^{2} \rightarrow$ $\mathbb{C}^{2}$ be a homogeneous polynomial map of degree $\geq 2$ with $\Phi^{-1}(0)=\{0\}$, and let

$$
\Omega=\left\{z \in \mathbb{C}^{2}: \Phi^{\circ n}(z) \rightarrow 0\right\} .
$$

Since $\Phi$ maps complex lines through the origin to complex lines through the origin, $\Phi$ induces a holomorphic self-map $\phi=\Phi_{1} / \Phi_{2}$ of $\mathbb{C} P^{1}=\hat{\mathbb{C}}$.

Theorem 2.5 (Hubbard-Papadopol [HuPa, Section 7]) $\Omega$ is a complete circular domain, and the corresponding Levi measure on $\hat{\mathbb{C}}$ is $4 \pi$ times the Brolin measure associated to the rational map $\phi$. In particular, the Levi measure is supported on the Julia set of $\phi$.

Thus $\Omega$ will satisfy the hypotheses of Theorems 1.2, 1.4, and 1.5 if the Julia set has vanishing one-dimensional Hausdorff measure; this happens for example if $\Phi\left(z_{1}, z_{2}\right)=\left(z_{1}^{2}+c z_{2}^{2}, z_{2}^{2}\right),|c| \geq 4$ [Ran, Ex. 2].

On the other hand, if we take $\Phi\left(z_{1}, z_{2}\right)=\left(z_{1}^{2}, z_{2}^{2}\right)$ then $\Omega$ is the bidisk discussed in Example 2.1.

Proof. [Sketch of proof that $K \subset$ Julia set] After establishing that

$$
\begin{aligned}
r(\zeta) & =\lim _{n \rightarrow \infty} \sqrt{1+|\zeta|^{2}}\left\|\Phi^{\circ n}(\zeta, 1)\right\|^{k^{-n}} \\
& =\lim _{n \rightarrow \infty} \sqrt{1+|\zeta|^{2}}\left|\Phi_{2}^{\circ n}(\zeta, 1)\right|^{k^{-n}}\left(\sqrt{1+\left|\phi^{\circ n}(\zeta)\right|^{2}}\right)^{k^{-n}}
\end{aligned}
$$


we have (with suitable adjustments at zeros of $\Phi_{2}$ )

$$
\begin{aligned}
& d * d \log r \\
= & \lim _{n \rightarrow \infty}\left\{d * d \log \sqrt{1+|\zeta|^{2}}+k^{-n} d * d \log \left|\Phi_{2}^{\circ n}(\zeta, 1)\right|\right. \\
& \left.+k^{-n} d * d \log \sqrt{1+\left|\phi^{\circ n}(\zeta)\right|^{2}}\right\} \\
= & \omega+0+k^{-n} \lim _{n \rightarrow \infty}\left(\phi^{\circ n}\right)^{*} \omega .
\end{aligned}
$$

Since the $\left(\phi^{\circ n}\right)^{*} \omega$ are uniformly bounded off of the Julia set, the result now follows from Proposition 2.3 and the pseudoconvexity of $\Omega$.

Remark 2.6 See [HuPa] for discussion of the higher-dimensional situation.

An earlier construction (by potential-theoretic means) of domains satisfying Theorems 1.2, 1.4, and 1.5 is due to Sibony [Sib].

3. Extension from $P$. The proofs of Theorems 1.1 and 1.2 will be based on the Cauchy integral

$$
\mathcal{C} f(z) \stackrel{\text { def }}{=} \frac{1}{2 \pi i} \int_{\mathrm{b} \Omega_{z_{1} / z_{2}}} \frac{f(w) d w_{1}}{w_{1}-z_{1}}=\frac{1}{2 \pi i} \int_{\mathrm{b} \Omega_{z_{1} / z_{2}}} \frac{f(w) d w_{2}}{w_{2}-z_{2}} .
$$

For $\zeta_{0} \notin K$ we can find parameterizations $w(\zeta, t)$ of $\mathrm{b} \Omega_{\zeta}$ for $\zeta$ near $\zeta_{0}$ varying holomorphically with $\zeta$. Thus,

$$
\mathcal{C} f(z)=\frac{1}{2 \pi i} \int_{0}^{2 \pi} \frac{f\left(w\left(\frac{z_{1}}{z_{2}}, t\right)\right) \frac{\partial w_{j}}{\partial t}\left(\frac{z_{1}}{z_{2}}, t\right)}{w_{j}\left(\frac{z_{1}}{z_{2}}, t\right)-z_{j}} d t
$$

is holomorphic on

$$
U \stackrel{\text { def }}{=}\left\{z: z \neq 0, \frac{z_{1}}{z_{2}} \notin K\right\} \backslash \mathrm{b} \Omega
$$

when $f \in H^{\infty}(P)$.

Let $U_{+}, U_{-}$denote the bounded and unbounded components of $U$, respectively, and denote the restrictions of $\mathcal{C} f$ to $U_{ \pm}$by $\mathcal{C}_{ \pm} f$. Also let

$$
P^{*} \stackrel{\text { def }}{=} P \cap\left\{z: \frac{z_{1}}{z_{2}} \notin K\right\} \text {. }
$$

Then the $\mathcal{C}_{ \pm} f$ have non-tangential boundary values $\mathcal{C}_{ \pm}^{*} f \in \operatorname{BMO}\left(P^{*}\right)$ and

$$
f=\mathcal{C}_{+}^{*} f-\mathcal{C}_{-}^{*} f .
$$


(See for example [Chr, Thms. IV.10, III.4], [Mar, Vol. 1, Thm. 14.12].)

Note also that

$$
\lim _{\|z\| \rightarrow \infty} \mathcal{C}_{-} f(z) \rightarrow 0
$$

on $U_{-}$.

Proof. [Proof of Theorem 1.2] The boundedness of $f$ and the standing assumption that

$$
\sup _{\zeta} \Lambda_{1}\left(\mathrm{~b} \Omega_{\zeta}\right)<\infty
$$

imply that $\mathcal{C} f$ is bounded on $U \cap L$, where $L$ is any compact subset of $\mathbb{C}^{2} \backslash \mathrm{b} \Omega$. Now Painlevé's Theorem [Gar, p. 1 and Thm. I.1.4] implies that $\mathcal{C}_{+} f$ and $\mathcal{C}_{-} f$ extend holomorphically along each horizontal and each vertical line in $\Omega \backslash\{0\}$ and $\mathbb{C}^{2} \backslash \bar{\Omega}$, respectively. Since the extensions are given by Cauchy integrals, we find that $\mathcal{C}_{+} f$ and $\mathcal{C}_{-} f$ in fact extend holomorphically to $\Omega \backslash\{0\}$ and $\mathbb{C}^{2} \backslash \bar{\Omega}$.

By Hartogs' Extension Theorem [Hör, Thm. 2.3.2], $\mathcal{C}_{+} f$ and $\mathcal{C}_{-} f$ further extend holomorphically to $\Omega$ and $\mathbb{C}^{2}$. But the extended $\mathcal{C}_{-} f$ now satisfies (3.5) on all of $\mathbb{C}^{2}$ so that Liouville's Theorem forces $\mathcal{C}_{-} f$ and hence $\mathcal{C}_{-}^{*} f$ to vanish identically.

It follows now that $\mathcal{C}_{+} f$ can also be obtained by taking Poisson integrals of $f$ along slices and hence that $\left\|\mathcal{C}_{+} f\right\|_{L^{\infty}(\Omega)}=\|f\|_{L^{\infty}(P)}$.

Proof. [Proof of Theorem 1.1] Hypotheses (1) and (2) imply that (3.3) defines a continuous function $\mathcal{C} f$ on $\mathbb{C}^{2} \backslash \mathrm{b} \Omega$ which is holomorphic on $U$. By [Gar, III.2.4] $\mathcal{C}_{+} f$ and $\mathcal{C}_{-} f$ are holomorphic along each horizontal and each vertical line in $\Omega \backslash\{0\}$ and $\mathbb{C}^{2} \backslash \bar{\Omega}$, respectively. By [Hör, Thm. 2.2.1] $\mathcal{C}_{+} f$ and $\mathcal{C}_{-} f$ are holomorphic in $\Omega \backslash\{0\}$ and $\mathbb{C}^{2} \backslash \bar{\Omega}$. The argument from the preceding proof again forces $\mathcal{C}_{-}^{*} f \equiv 0$, so that once again $\mathcal{C}_{+} f$ can be obtained by taking Poisson integrals of $f$ along slices. Standard estimates on harmonic measure [Tsu, Thm. III.67] suffice to show that $f$ and $\mathcal{C}_{+} f$ together define a function in $A(\Omega)$.

4. Leaves. Proof. [Proof of Theorem 1.3] Suppose to the contrary that some leaf $L$ is closed in $P$. Then

$$
\begin{aligned}
\rho: L^{*} \stackrel{\text { def }}{=} L \cap P^{*} & \rightarrow \hat{\mathbb{C}} \backslash K \\
z & \mapsto z_{1} / z_{2}
\end{aligned}
$$

is a proper holomorphic map of Riemann surfaces. 
Fix $\zeta_{0} \in \mathbb{C} \backslash K$ and let $p$ be a polynomial in $z_{2}$ vanishing at 0 and $\equiv 1$ on $\rho^{-1}\left(\zeta_{0}\right)$. Introduce

$$
q(\zeta)=\sum_{z \in \rho^{-1}(\zeta)} p\left(z_{2}\right)
$$

on $\hat{\mathbb{C}} \backslash K$. (Multiplicities are to be respected in the sum.) $q$ is bounded and holomorphic, hence constant by Painlevé's Theorem. But this contradicts $q\left(\zeta_{0}\right)=\#\left(\rho^{-1}\left(\zeta_{0}\right)\right)>0, \lim _{\zeta \rightarrow \infty} q(\zeta)=0$.

Remark 4.1 The map $\rho$ in fact has no branch points and is thus a covering map. To see that $\rho$ is unbranched at some fixed $z \in L$, choose a local holomorphic defining function $h$ for $L$ at $z$ so that $P$ admits a local defining function $\sigma$ of the form $\sigma=\operatorname{Re} h+o\left(|h|^{2}\right)$ [BaFo, Prop.]. Then if $L$ has a radial tangency of order $k$ at $z$, restriction of $\sigma$ to the line joining 0 to $z$ reveals that $\mathrm{b} \Omega_{z_{1} / z_{2}}$ has a vertex of order $2 k+2$ at $z$, contrary to the assumption that $\mathrm{b} \Omega_{z_{1} / z_{2}}$ is a Jordan curve.

Proof. [Proof of Theorem 1.4] From Example 2 we carry over the notation $\chi$, $\widetilde{D}_{\text {Hom }}$.

Also, for $\tau_{0} \in \mathbb{T}$ let

$$
\begin{aligned}
R_{\tau_{0}}: \mathbb{T} & \rightarrow \mathbb{T} \\
\tau & \mapsto \tau_{0} \cdot \tau
\end{aligned}
$$

denote rotation by $\tau_{0}$.

Note that the hypothesis $\Lambda_{1}(K)=0$ implies that $\hat{\mathbb{C}} \backslash K$ is connected and hence coincides with $D$.

Let $\Gamma$ denote the subgroup $\chi\left(H^{1}(D)\right)$ of $\mathbb{T}$. By Theorem 1.3 and the description (2.2) of the deck groups, $\Gamma$ is infinite and thus dense in $\mathbb{T}$.

Lemma 4.2 We can find generators $\gamma_{1}, \gamma_{2}, \ldots$ of $\Gamma$ and measurable maps

$$
\nu_{d}: \mathbb{T} \rightarrow \Gamma
$$

such that

(1) $\left\|\nu_{d}-I_{\mathbb{T}}\right\|_{L^{\infty}(\mathbb{T})}<2^{-d}$;

(2) for all $j=1, \ldots$, d there is $\tau_{j, d} \in \mathbb{T}$ such that $\left|\tau_{j, d}-\gamma_{j}\right|<2^{-d}$ and

$$
\left|\left\{\tau \in \mathbb{T}: \nu_{d} \circ R_{\tau_{j, d}}(\tau) \neq R_{\gamma_{j}} \circ \nu_{d}(\tau)\right\}\right|<2^{-d} .
$$


We defer the proof of Lemma 4.2 to the end of the section.

To complete the proof of Theorem 1.4 we introduce

$$
\begin{aligned}
E_{d}: L^{\infty}\left(\widetilde{D}_{\mathrm{Hom}} \times \Gamma\right) & \rightarrow L^{\infty}\left(\widetilde{D}_{\mathrm{Hom}} \times \mathbb{T}\right) \\
f & \mapsto f \circ\left(I_{\widetilde{D}_{\mathrm{Hom}}} \times \nu_{d}\right) .
\end{aligned}
$$

(Here $\Gamma$ is assigned counting measure.) Note that

$$
\begin{gathered}
\left\|E_{d}\right\|=1, \\
E_{d}(f g)=E_{d}(f) \cdot E_{d}(g),
\end{gathered}
$$

and

$$
E_{d}\left(H^{\infty}\left(\widetilde{D}_{\mathrm{Hom}} \times \Gamma\right)\right) \subset H^{\infty}\left(\widetilde{D}_{\mathrm{Hom}} \times \mathbb{T}\right) .
$$

By the Banach-Alaoglu Theorem the operators $E_{d}$ have an accumulation point

$$
E_{\infty}: L^{\infty}\left(\widetilde{D}_{\mathrm{Hom}} \times \Gamma\right) \rightarrow L^{\infty}\left(\widetilde{D}_{\mathrm{Hom}} \times \mathbb{T}\right)
$$

in the weak* topology on

$$
L^{\infty}\left(\widetilde{D}_{\text {Hom }} \times \Gamma\right) \otimes L^{1}\left(\widetilde{D}_{\text {Hom }} \times \mathbb{T}\right)
$$

We have

$$
\begin{gathered}
\left\|E_{\infty}\right\|=1 \\
E_{\infty}(f g)=E_{\infty}(f) \cdot E_{\infty}(g)
\end{gathered}
$$

and

$$
E_{\infty}\left(H^{\infty}\left(\widetilde{D}_{\mathrm{Hom}} \times \Gamma\right)\right) \subset H^{\infty}\left(\widetilde{D}_{\mathrm{Hom}} \times \mathbb{T}\right)
$$

Item (1) of Lemma 4.2 implies that

$$
E_{\infty}\left(\left.f\right|_{\widetilde{D}_{\text {Ноm }} \times \Gamma}\right) \equiv f \text { for } f \in C\left(\widetilde{D}_{\text {Hom }} \times \mathbb{T}\right) \cap L^{\infty}\left(\widetilde{D}_{\text {Hom }} \times \mathbb{T}\right) .
$$

Moreover, from item (2) of Lemma 4.2 and the weak * convergence we find that

$$
E_{\infty}\left(f \circ R_{\gamma}\right) \equiv\left(E_{\infty} f\right) \circ R_{\gamma} \text { almost everywhere on } \widetilde{D}_{\text {Hom }} \times \mathbb{T}
$$

for all $\gamma \in\left\{\gamma_{1}, \gamma_{2}, \ldots\right\}$, hence for all $\gamma \in \Gamma$. Thus using (2.1) and (2.2) to pass to quotients, we find that $E_{\infty}$ induces an operator

$$
E^{\prime}: L^{\infty}(L) \rightarrow L^{\infty}(P) .
$$


Evidently we have

$$
\begin{gathered}
\left\|E^{\prime}\right\|=1, \\
E^{\prime}(f g)=E^{\prime}(f) \cdot E^{\prime}(g), \\
E^{\prime}\left(H^{\infty}(L)\right) \subset H^{\infty}(P),
\end{gathered}
$$

and

$$
E^{\prime}\left(\left.f\right|_{L}\right)=f \text { for } f \in C(P) \cap L^{\infty}(P) .
$$

The desired operator $E$ is now constructed by composing $E^{\prime}$ with the extension operator

$$
H^{\infty}(P) \rightarrow H^{\infty}(\Omega)
$$

from Theorem 1.2.

Proof. [Proof of Theorem 1.5] Letting $z_{1}, z_{2}$ denote the coordinate functions on $\mathbb{C}^{2}$ we have

$$
\iota_{L}(L)\left(z_{1}, z_{2}\right)=L
$$

so that

$$
\overline{\iota_{L}(L)}\left(z_{1}, z_{2}\right) \subset \bar{L}=\mathrm{b} \Omega
$$

whereas

$$
\hat{E}\left(\iota_{\Omega}(\Omega)\right)\left(z_{1}, z_{2}\right)=\Omega .
$$

Thus $E\left(\iota_{\Omega}(\Omega)\right) \cap \overline{\iota_{L}(L)}=\varnothing$.

Proof. [Proof of Lemma 4.2]

Case 1: $\Gamma$ consists of roots of unity. Since $\Gamma$ is dense, we may choose generators $\gamma_{1}, \gamma_{2}, \ldots$ in the first quadrant approaching 1 monotonically so that $\gamma_{j+1}^{n_{j}}=\gamma_{j}$ for some $n_{j}>1$. For $\tau \in \mathbb{T}$ let $\nu_{d}(\tau)$ denote the first power of $\gamma_{d}$ lying clockwise from $\tau$. (We set $\nu_{d}\left(\gamma_{d}^{n}\right)=\gamma_{d}^{n}$.) Then conclusion (1) follows easily from $\arg \left(\gamma_{d}\right)<2^{-d}$, and for conclusion (2) we set $\tau_{j, d}=\gamma_{j}$ and note that $\nu_{d} \circ R_{\gamma_{j}} \equiv R_{\gamma_{j}} \circ \nu_{d}$.

Case 2: $\Gamma$ has elements of infinite order. For this case it will suffice to prove the following: 
( $\star$ If $\Gamma$ is a finitely-generated infinite subgroup of $\mathbb{T}$ and $S$ is a finite subset of $\Gamma$, then for each $\varepsilon>0$ we may find a measurable map $\nu: \mathbb{T} \rightarrow \Gamma$ such that

(1) $\left\|\nu-I_{\mathbb{T}}\right\|_{L^{\infty}(\mathbb{T})}<\varepsilon$;

(2) for all $\gamma \in S$ there is $\tau_{\gamma} \in \mathbb{T}$ such that $\left|\tau_{\gamma}-\gamma\right|<\varepsilon$ and

$$
\left|\left\{\tau \in \mathbb{T}: \nu_{d} \circ R_{\tau_{\gamma}}(\tau) \neq R_{\gamma} \circ \nu_{d}(\tau)\right\}\right|<\varepsilon .
$$

Such a group $\Gamma$ is isomorphic to $\mathbb{Z}_{q_{0}} \times \mathbb{Z}^{d}$ [Rot, Thm. 9.26]; it will suffice to prove $(\star)$ under the assumption that $S$ is the set of generators $\left\{\gamma_{0}, \gamma_{1}, \ldots, \gamma_{d}\right\}$ corresponding to this decomposition. We may assume that $\gamma_{j}=e^{2 \pi i \theta_{j}}$ with $0 \leq \theta_{0} \leq \frac{1}{2}, 0<\theta_{j}<\frac{1}{2}$ for $j \geq 1$.

$\theta_{0}$ is of course a rational number $p_{0} / q_{0}$. By the theory of continued fractions (see for example [Arn, Section 11.L]), for each $j \geq 1$ we may find infinitely many rational approximations $p_{j} / q_{j}$ to the irrational number $\theta_{j}$ with $\left|\theta_{j}-p_{j} / q_{j}\right|<$ $1 / q_{j}^{2}$. (All fractions given in lowest terms.)

Let $s_{j}=\operatorname{lcm}\left\{q_{0}, q_{1}, \ldots, q_{j}\right\}$ and $r_{j}=\operatorname{gcd}\left\{s_{j-1}, q_{j}\right\} ;$ thus $s_{0}=q_{0}$ and $s_{j}=$ $\operatorname{lcm}\left\{s_{j-1}, q_{j}\right\}=s_{j-1} q_{j} / r_{j}$ for $j \geq 1$. By induction we may assume that $q_{j} / r_{j} \geq$ $q_{j} / s_{j-1}>4 \pi d / \varepsilon$ for $j \geq 1$.

Let $H$ denote the group of $s_{d}^{\text {th }}$ roots of unity. Define a map $\mu: \mathbb{T} \rightarrow H$ by letting $\mu(\tau)$ be the first point in $H$ lying clockwise from $\tau$. Note that $\| \mu-$ $I_{\mathbb{T}} \|_{L^{\infty}(\mathbb{T})} \leq 2 \pi / s_{d}<\varepsilon / 2$ and that $\tau \in H$ implies $\mu \circ R_{\tau} \equiv R_{\tau} \circ \mu$.

The rule

$$
\psi\left(\exp \left(2 \pi i\left(\frac{n_{0} p_{0}}{q_{0}}+\frac{n_{1} p_{1}}{q_{1}}+\cdots+\frac{n_{d} p_{d}}{q_{d}}\right)\right)\right)=\gamma_{0}^{n_{0}} \gamma_{1}^{n_{1}} \cdots \gamma_{d}^{n_{d}}
$$

defines a bijective map $\psi$ from $H$ to

$$
\left\{\gamma_{0}^{n_{0}} \gamma_{1}^{n_{1}} \cdots \gamma_{d}^{n_{d}}: 0 \leq n_{0}<q_{0}, 0 \leq n_{j}<\frac{q_{j}}{r_{j}} \text { for } j \geq 1\right\} \subset \Gamma .
$$

We will prove the Lemma by showing that $\psi$ is "quasi-homomorphic" and is metrically close to the identity.

We have

$$
\begin{aligned}
|\psi(\tau)-\tau| & <2 \pi \sum_{j=1}^{d} \frac{n_{j}}{q_{j}^{2}} \\
& <2 \pi \sum_{j=1}^{d} \frac{1}{q_{j}} \\
& <\frac{\varepsilon}{2}
\end{aligned}
$$


so that $\nu \stackrel{\text { def }}{=} \psi \circ \mu$ satisfies

$$
\begin{aligned}
\left\|\nu-I_{\mathbb{T}}\right\|_{L^{\infty}(\mathbb{T})} & \leq\|\nu-\mu\|_{L^{\infty}(\mathbb{T})}+\left\|\mu-I_{\mathbb{T}}\right\|_{L^{\infty}(\mathbb{T})} \\
& <\frac{\varepsilon}{2}+\frac{\varepsilon}{2}=\varepsilon .
\end{aligned}
$$

and thus $\nu$ satisfies condition (1) of $(\star)$.

Let $\tau_{\gamma_{j}}=\psi^{-1}\left(\gamma_{j}\right)$. Then

$$
\left|\tau_{\gamma_{j}}-\gamma_{j}\right|=\left|\tau_{\gamma_{j}}-\psi\left(\tau_{\gamma_{j}}\right)\right|<\varepsilon .
$$

For $j=0$ we have $\tau_{\gamma_{0}}=\gamma_{0}$ and $\psi\left(\gamma_{0} \tau\right)=\gamma_{0} \psi(\tau)$ for all $\tau \in H$, so that

$$
R_{\gamma_{0}} \circ \psi \circ \mu \equiv \psi \circ R_{\gamma_{0}} \circ \mu \equiv \psi \circ \mu \circ R_{\gamma_{0}} .
$$

For $j \geq 1$ we have

$$
\begin{aligned}
\psi\left(\tau _ { \gamma _ { j } } \operatorname { e x p } \left(2 \pi i \left(\frac{n_{0} p_{0}}{q_{0}}\right.\right.\right. & \left.\left.\left.+\frac{n_{1} p_{1}}{q_{1}}+\cdots+\frac{n_{d} p_{d}}{q_{d}}\right)\right)\right) \\
& =\gamma_{j} \psi\left(\exp \left(2 \pi i\left(\frac{n_{0} p_{0}}{q_{0}}+\frac{n_{1} p_{1}}{q_{1}}+\cdots+\frac{n_{d} p_{d}}{q_{d}}\right)\right)\right)
\end{aligned}
$$

except when $n_{j}=q_{j} / r_{j}-1$. Thus

$$
\frac{\#\left\{\tau \in H: \psi\left(\tau_{\gamma_{j}} \tau\right) \neq \gamma_{j} \psi(\tau)\right\}}{\# H}=\frac{1}{q_{j} / r_{j}}<\frac{\varepsilon}{2 \pi}
$$

and

$$
\left|\left\{\tau \in \mathbb{T}: R_{\gamma_{j}} \circ \psi \circ \mu \neq \psi \circ R_{\tau_{\gamma_{j}}} \circ \mu\right\}\right|<\varepsilon .
$$

Since $R_{\tau_{\gamma_{j}}} \circ \mu \equiv \mu \circ R_{\tau_{\gamma_{j}}}$ we see that $\nu$ also satisfies condition (2) of ( $\star$ ).

Remark 4.3 Higher-dimensional analogues of Theorems 1.1, 1.2, 1.3, and 1.4 can be formulated by imposing suitable removable-singularity conditions on $K \subset \mathbb{C} P^{n-1}$.

\section{REFERENCES}

[Arn] V. I. Arnold. Geometrical Methods in the Theory of Ordinary Differential Equations, 2nd ed., Springer-Verlag, 1988.

[BaDi] D. BARrett And J. Diller. A new construction of Riemann surfaces with corona, J. Geometric Analysis, to appear.

[BaFo] D. BARrett And J. E. Fornæss. On the smoothness of Levi-foliations, Pub. Math. UAB (Barcelona) 32 (1988), 171-177.

[BG] E. BEDFord AND B. Gaveau. Hypersurfaces with bounded Levi form, Indiana Univ. Math. J. 27 (1978), 867-873. 
[Car] L. Carleson. Selected Problems on Exceptional Sets, D. van Nostrand Co., 1967.

[Chr] M. Christ. Lectures on Singular Integral Operators, CBMS Regional Conf. Series in Math., Vol. 77, Amer. Math. Soc., 1990.

[Fal] K. J. Falconer. The Geometry of Fractal Sets, Cambridge University Press, 1985.

[For] O. Forster. Lecture on Riemann Surfaces, Springer-Verlag, 1981.

[Gar] J. Garnetr. Analytic Capacity and Measure, Lecture Notes in Mathematics, Vol. 297, Springer-Verlag, 1972.

[Hör] L. Hörmander. An Introduction to Complex Analysis in Several Variables, 3rd ed., North-Holland, 1990.

[HuPa $]$ J. H. Hubbard and P. Papadopol. Superattractive fixed points in $\mathbb{C}^{n}$, Indiana Univ. Math. J. 43 (1994), 321-365.

[Mar] A. I. Markushevich. Theory of Functions of a Complex Variable, 2nd ed., Chelsea Publishing Co., 1965.

[Mor] F. Morgan. Geometric Measure Theory: A Beginner's Guide, Academic Press, 1988.

[Ran] T. J. RANSFord. Variation of Hausdorff dimension of Julia sets, Ergodic Theory and Dynamical Systems 13 (1993), 167-179.

[Rot] J. Rotman. Theory of Groups, Allyn and Bacon, 1965.

[Sib] N. Sibony. Sur la frontière de Shilov des domaines de $\mathbb{C}^{n}$, Math. Ann. 273 (1985), 115-121.

[Tsu] M. Tsuji. Potential Theory in Modern Function Theory, Maruzen, 1959.

This research was partially supported by a grant from the National Science Foundation.

Department of Mathematics

The University of Michigan

Ann Arbor, Michigan 48109-1003

E-MAIL: barrett@umich.edu

Received: April 26th, 1995. 